\title{
AN ARCHAEOLOGICAL STUDY OF THE RUSSIAN COLONY OF THE GUADALUPE VALLEY
}

\author{
By \\ Therese Muranaka*
}

\begin{abstract}
This fresh and unusual archreological study sets out to verify the accuracy with which athnological data and facts regarding life-style may be determined by the examination of artifacts and "trash" found at the site, using the application of archeoological techniques in an area where the findings may still be corroborated or negated by living informants. The investigation site is the Molokan Colony of the Guadalupe Valley of the State of Baja Califomia, Mexico.
\end{abstract}

\section{RESUMEN}

Este estudio arqueológico, fresco y fuera de lo común, pretende mirar la autenticidad con la cual los datos etnológicos y revelaciones del estilo de vida de una comuniciad se pueden determinar exmminando los artefactos y "besura" encontrados en el sitio, mediante la aplicacion de técnicas arqueologicas en un área donde las respuestas todavía pueden ser corroboradas o negadas por los primeros habitantes. El lugar de investigación es la colonia rusa Molokan del valle de Guadalupe, en el estado de Baja Califomia, México.

\section{INTRODUCTION ${ }^{1}$}

As a foreign student of archaeology in 1975 and 1976 in the East European city of Bucharest, I was overwhelmed at first by the diversity of speech, faces and clothing. I remember taking tram rides through the old city, observing Macedonians, Hungarians, Jews, and Gypsies interacting with each other in polite but limited conversations. I remember having a conversation with a Romanian employee at an embassy library. Although a very unassuming woman, she wore a garish silver bracelet with swirled engravings that contrasted with her professional image. When I asked her

- Director of the Serra Museum, San Diego, Califomia.

1 Special thanks to Julie Bendimez-Patterson of INAH, Baja California; George Mohoff, the exceptional Molokan historian; Molokan John J. Samarin of Los Angeles; Katherine Abukumoff of the Usicn in Los Angeles for her map; my translator, Elena Teress Orozco, who was also a descendent of the Guadalupe Valley. Special thanks go also to the people of the Guadalupe Valley, who, although tired of being picked and poked by anthropologists, archacologists and historians, but who always responded with patience. I would also like to thank my husband Jason and my litule son, Jay-Michael. 
about the bracelet, she smiled and said her grandmother had been captured by pirates as a child and taken to a Black Sea market. Purchased and raised by a Romanian family, the grandmother had no memory of where she was born, except the bracelet that she wore on her wrist. Her granddaughter always wore this piece of material culture, a clue to the family's ethnicity, in the hopes that someday someone would recognize it. It was her dream that someone would notice the style, the quality, or how the silver was worked and tell her who she was.

That was why I was there, to track migrations thousands of years old by the material objects which the people had left behind, but I quickly became confused; migration $1 \mathrm{~A}$ preceded migration $\mathrm{BB}$, which preceded migration $2 \mathrm{~A}$ and $2 \mathrm{~B}$, as confused archeologists attempted to sort through thousands of years of the movements of peoples. Challenged by the vast data base, I came back to San Diego and proceeded to sift through the literature on prehistoric migrations and how to identify them. I reasoned "If I could just find a recent migration of a unique people, I could trace it, and see how migration works". If the migrants or their descendents were still alive I could even check with them as to the accuracy of my reconstruction of their former lives. But where? Who? And then the answer came to me: the Russian Molokans of the Guadalupe Valley in nearby Baja California (Figure 1). They were a relatively recent migration (1904-1912) to a region which formerly had been populated by prehistoric Indian peoples, followed by Spanish missionaries, and later, Mexicans on ranchos and in port towns. And there begins the tale of a ten-year research design working with the Molokans of the Guadalupe Valley.

\section{THE MOLOKANS}

Published elsewhere (Muranaka, 1987:125-135), the history of the Russian Molokans will only be summarized here. They are the Molokanye or "Milk Drinkers", religious dissenters who were formed from a parent sect known as the Doukhobors sometime after 1760 (Stepniak, 1888:323). They were perhaps called "those who drink milk" because they drank milk at times when their Russian Orthodox neighbors could not, for example, during the Lenten season fasts. Another explanation given for their name is that because they ate kosher style like the Orthodox Jews, they could only drink the milk of a prison meal, for fear it might be contaminated with pork or some other unclean food. A third explanation is that they had originally come from the Molotchnaye or "Milky" River region of Russia. They also have two other names: "Spiritual Christians" and the "Jumpers" (because they "jump" in ecstasy during their religious services). 


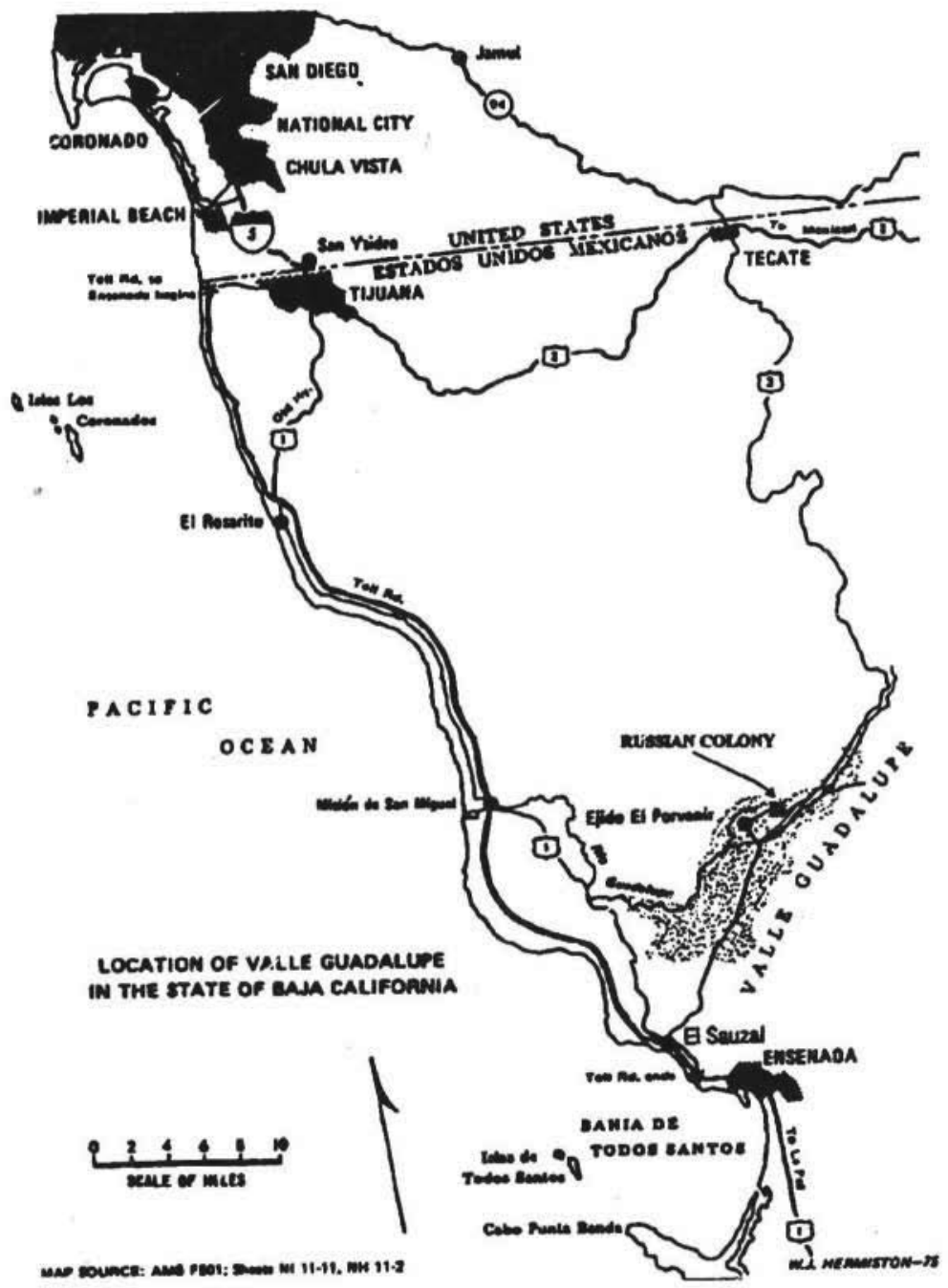

Figure 1. Map of Northern Baja California, Showing Location of the Guadalupe Valley and the Russian Colony. 
The Russian Molokans are Christians, although they keep Kosher as do Jews, but do not believe in priests, sacraments, hierarchical church organizations, higher and lower status among people, or in any ownership or display of material wealth. Simple in dress, speech and material culture. this pacifist sect spread from Tambov in Central Russia to the Caucasus Mountains (Figure 2). In 1839 a fifty-year exemption from the draft into the Russian army was offered them if they would settle in the isolated parts of what is today Armenia and far eastern Turkey. Beginning in 1855, a small boy-prophet began telling tales of terrible times of trial to came. By 1899 , with the emigration of their sister sect, the Doukhobors, to Cansda, the Molokans had decided the time had come to leave Russia (see Mohoff, 1992a for the best discussion). Some say with admiration and belief that the child told them they would be protected anywhere in the world if they just stayed with the angels, and some say that it was at the urging of the famous Russian writer Leo Tolstoy's literary agent in the United States, that they came to arrive by land and by sea at the port of Los Angeles, the "Port of Angels", in Alta California by 1905-1906. Whatever may be the true explanation, during the time of Porfirio Diaz, 104 of these families purchased the 13,000-acre ex Mision Nuestra Sellora de Guadalupe on the Río Guadalupe north of Ensenada, approximately 35 miles southeast of Tijuana.

The approximately 900 -member colony was laid out within a few short years on the former lands of the 1834 Dominican mission, a large parcel, the ownership of which had then passed to 19th-century rancher Jun Bandini, and had been the outpost of the jefe polftico of northern Baja California, José Matfas Moreno (Long, 1972). The colony lastod undisturbed until the first major emigration in 1912. Dissatisfaction with the difficulties of farming, and fear of uprising by Villistas against foreign investors (even as far west as Baja California) are the reasons given for the departure of some of the original colonists. In 1937, President Lázaro Cárdenas reapportioned single large tracts of land in the ejido movement and the formation of the Ejido Porvenir frightened mare Russian families into leaving. The final impact was the construction in 1958 of the Tecate-to-Ensenada road (Highway 3), and the increasingly high visibility of the fertile Russian farms. A group of 3006 workers appeared in the night (July 10, San Diego Union, $1958 \mathrm{a}-$ f and 1959) and occupied both Molokan and Mexican-owned farms in the valley. Called the Paracaidistas ("Parachutists") because they seemed to appear from the skies, they formed a poblado known as Francisco Zanco which gave impetus to the last exodus of Molokans from the valley. In 1996, few of the original Molokan ("pure Russians") remain. 


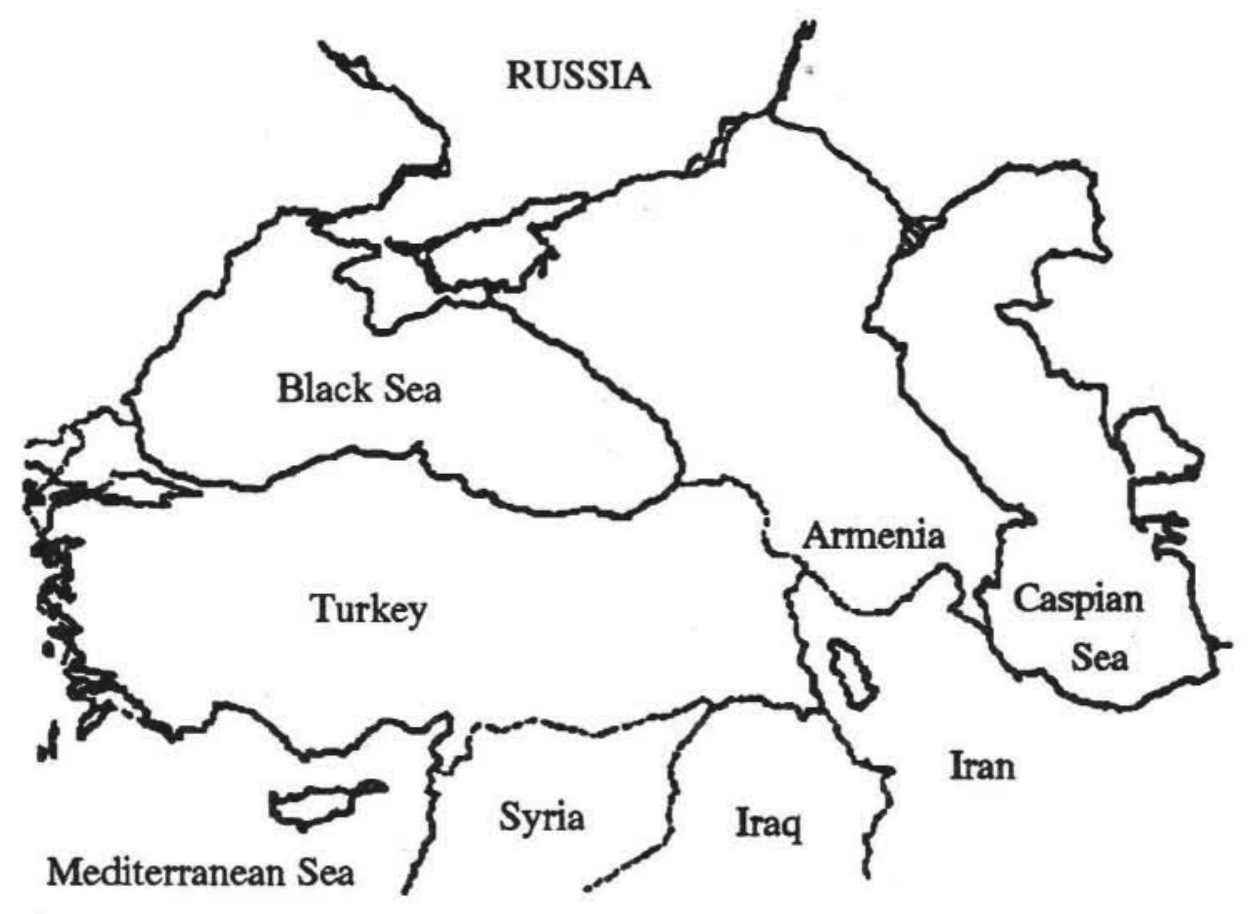

Figure 2. Map of Kars and Vicinity, Origin of Molokan Exodus to the United States at the Turn of the Centary. 


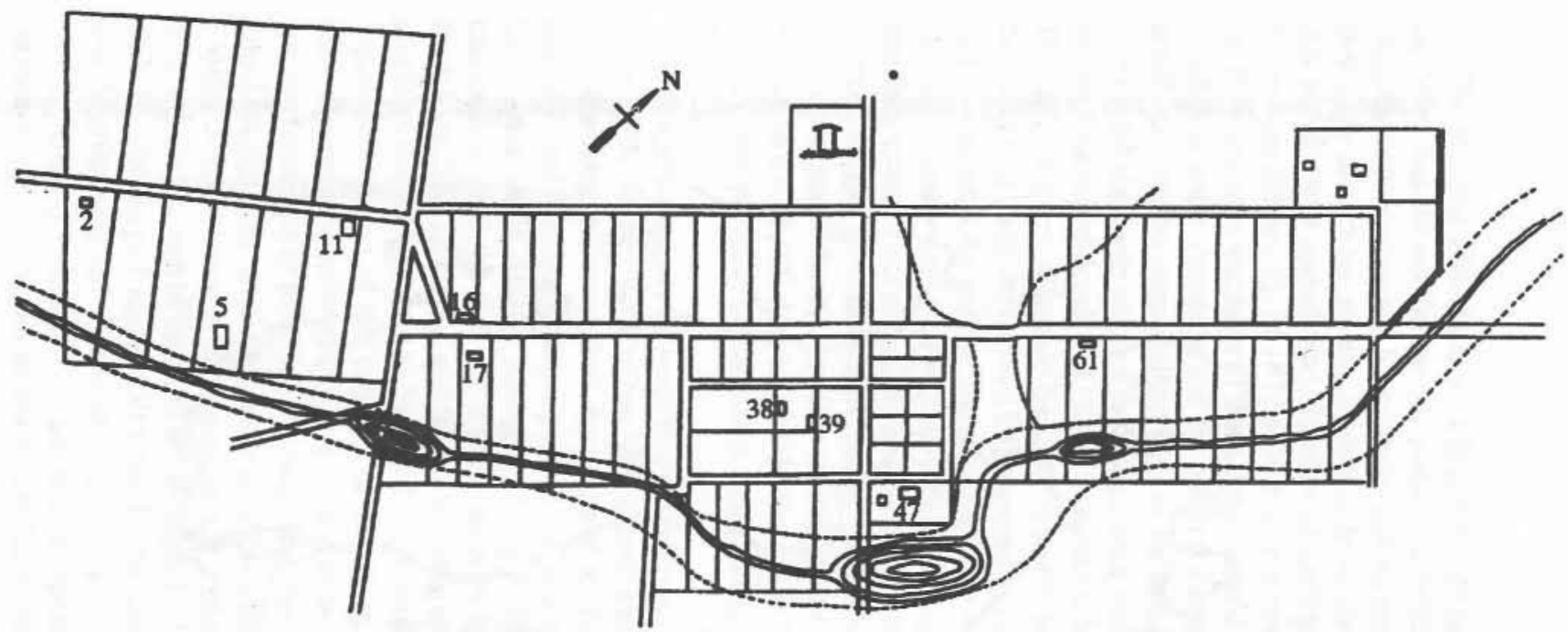

Structures 2 and 5 Grigari Danielich Afonin Structure 11. Moisés Grigorich Samarin

Structure 16 Juan Vasilich Rogoff

Structure 17 Ivan Pavlich Rogoff

Structure 39. Susan Ivanovna Kachiriski

Structure 47 Timofei M. Samarin Mission Ruins)

Figure 3Molokan Guadalupe Colony, 1905-1960.

Note: Modified from map in Mohoff (1992b).

Structure 61. Andres Moiseich Samaduroff 


\section{EXCAVATION HISTORY}

Once the colony had been selected, the process of obtaining anchaeological excavation permits was begun. With the most kind help of Ing. Joaquin Garcia-Bancena, then the Director of Prehistoric Monuments of the Instituto Nacional de Antropologia e Historia (INAH), Jaime Abundis of INAH, Mexico City, of Julie Bendímez Patterson of INAH, Baja California, and the late David Zarate-Loperena of Asuntos Culturales, Ensenada, B.C.. my husband and I obtained INAH permits to excavate the Molokan sector of Guadalupe within two weeks of our request in 1985.

We began digging in September of 1985, and for each of the five separate fieldwork sessions, I took with me Spanish-language permits from INAH's Office of Prehistoric Monuments in Mexico City, regional papers from INAH in Baja California, letters of introduction from the cultural anthropologist of the nearest large town, the municipio of Ensenada, stamps from the delegado of Francisco Zarco, and verbal permissions and interviews from each of the owners of the private parcels. With crews as large as 15 to 20, and made up of both Mexican and U.S. archaeologists and historians, we proceeded to carefully excavate balanced sample grids at seven separate houses (Figure 3). The task was to see if we could determine the ethnicity of the occupants, and then interview the descendents to see how accurate we were. Excavations took place September 27 to 29, and October 4 through 6, 1985; March 14 to 16, and 20 to 21, 1987; September 30, October 1 and 2,1989, and October 20 to 22, 1990, with many trips to the storage facility at David Zarate's house in Ensenada, where sorting, washing, cataloguing, photographic work, sketching and curating the artifacts took place. Approximately 89,000 artifacts were processed by Christmas Eve, 1991, with many months of follow-up to finish the final paperwork.

\section{EXCAVATION RESULTS}

For a summary of excavation history and test findings, it is best to proceed with test results structure by structure, starting with a short ethnography of each occupant.

\section{Structure 39. Susana Ivanovna Kachiriski}

The colony's Susana Ivanovna Kachiriski (standard Russian Susana Ivanovna Kachiriskaya, nee Potsakaieva) was born in Russia in 1897, the daughterof Ivan Polikarpovich and Marriya Potsakaiev. Sometime between 1916 and 1918, she married Vasili Kachiriski in the Guadalupe 
Colony. They moved to Los Angeles, Alta Califomia, and had five children. In the early 1930's, Susana's mother died, leaving a house (Figure 3, Str. 38) and property, including a large stock of cattle, to Susans. Susana and Vasili returned to Guadalupe, where one more child was born. Vasili returned to Los Angeles, leaving Susana to raise the children. One by one, reaching the age of 18, they returned to Los Angeles, where they were still U.S. citizens. According to George Mohoff, who was raised in the colony, Susana continued to live by herself in Guadalupe, and was the oldest of the Molokans bom in Russia. She supported herself by working as a maid, a laundress and a cook for families in the colony. She died about 1986.

I had heard of Susana on ethnographic field trips to Guadalupe between 1983 and 1985, but she died before I was able to interview her. When I entered the yard of her house a few months after her death, there were still scraps of her letters and shopping lists blowing around the yard. It was the most recent anchaeology I had ever done. A nephew, the son of her husband's ' brother and his Mexican wife, had taken occupancy of the house in recent weeks, and had planted a small garden. He said that the day Susana died, people had come from all over and scavenged everything, looking for the lost gold that everyone in the valley "knew" the Russians had hoarded.

Susana's yard (Figure 4) actually contained two structures: her own house (Structure 39) and the long-abandoned house of her parents (Structure 38), which had been repeatedly plowed over to make small truck gardens. Two test units were placed on March 13th, 14th, 20th and 21st, 1987 in the areas showing the most surface artifacts. Unit 1 cut through adobe melt and cobblestone foundations quickly, to find sterile soil at 80 $\mathrm{cm}$, and fewer than 200 items were catalogued, mainly unidentifiable metal, glass, and seeds, bone and wood. Unit 2 was a trash pit of burnt artifacts tallying 62,867 artifacts (Table 1 through 8 ). This $1 \times 2$ meter unit was $200 \mathrm{~cm}$. deep at sterile.

The collection dated from the late 1940's to the 1950's. Of the Russian-style glasses used for tea, 33 fragments were found, the largest number for any of the sites. Of the bottle glass, 376 (2738.3 grams) fragments were of clearly identifiable alcoholic beverages, which, though not allowed in the colony in the early years, became a creeping "vice" acceptable in later times. Bleach bottles were predominant (304 pieces) and food bottles were evident. Susana had worked as a maid and washer woman to support herself (hence the bleach bottles), and was paid in food, according to George Mohoff and Agáfia Rogoff. Medicine bottles numbered 19 fragments (258.3 grams), the largest number of all the sites. Noted were 26 fragments (884.1 grams) of cold cream jars. These were utilitarian varieties purchased in the United States and commonly used as dry skin aids. 


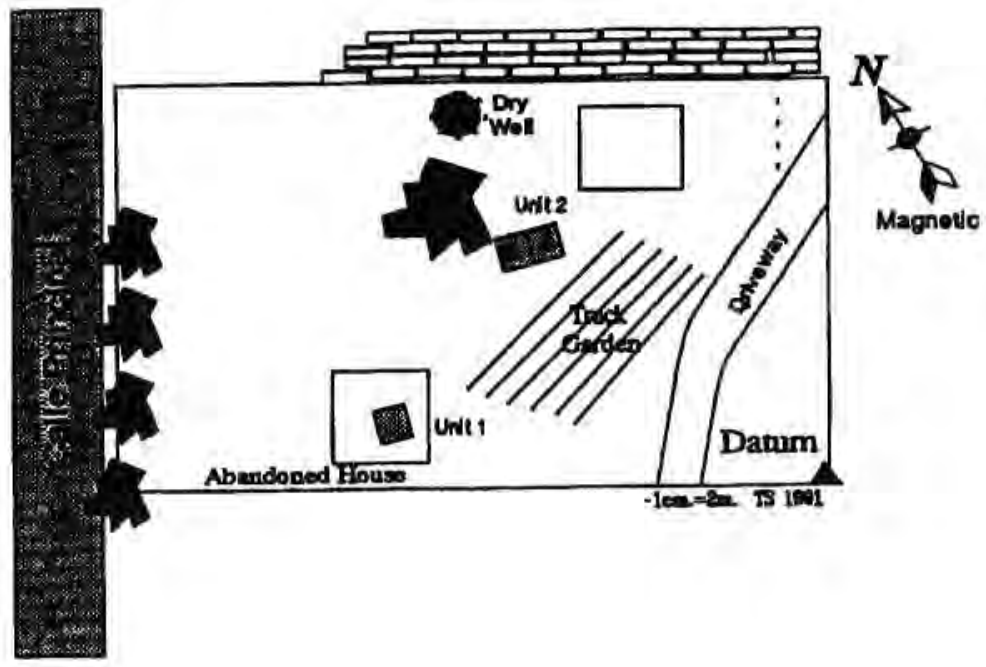

Figure 4. Plan of Susana Ivanovna Kachiriskd's House.

Susana showed a predilection (Tables 2 and 3) for attractively decorated, thin-walled porcelain, with floral or solid designs, and must have broken almost an entire floral set on one occasion, as six cups and saucers of a dainty peacock design ("Made in Japan") were dumped together. Cheaper designs of ironstone/whiteware (hotel ware) and inexpensive porcelains were in evidence. Cracked bowls, plates and cups sold in series from cereal boxes were also found. Molokans such as Mary and Agafia Rogoff expressed an aversion to "Chinese" ceramics. They associated the Chinese dragon motif with the devil, and avoided these patterns, especially the well-known "Blue Willow", with its pagoda and obvious Asian theme. Table 3 records all Chinese-made ware that as available from a colony of Chinese potters in Mexicali. Susana had this ware of Chinese make but with floral motif; she avoided the "Blue Willow" or any of the Chinese themes. 
Table 1. Guadalupe Glass by Structure.

\begin{tabular}{|c|c|c|c|c|c|c|c|c|}
\hline \multirow[b]{2}{*}{ Material } & \multirow[b]{2}{*}{ Sub-class } & \multirow[b]{2}{*}{ Object } & \multicolumn{2}{|c|}{ Russian } & \multicolumn{2}{|c|}{ Russian/Mexican } & \multicolumn{2}{|c|}{ in Mexican } \\
\hline & & & $\begin{array}{c}\text { str. } \\
39\end{array}$ & $\begin{array}{r}\text { str. } \\
61\end{array}$ & $\begin{array}{l}\text { str. } \\
11\end{array}$ & $\begin{array}{r}\text { str. } \\
16\end{array}$ & $\begin{array}{r}\text { str. } \\
2\end{array}$ & $\begin{array}{r}\text { str. } \\
76\end{array}$ \\
\hline \multirow{23}{*}{ Glass } & Bottle & Beer bottle & 160 & 0 & 1 & 174 & 74 & 110 \\
\hline & & Pop bottle & 13 & 0 & 141 & 10 & 11 & 61 \\
\hline & & Wine bottle & 8 & 0 & 0 & 0 & 0 & 0 \\
\hline & & Medicine & 19 & 0 & 0 & 12 & 13 & 0 \\
\hline & & Liquor & 208 & 0 & 0 & 746 & 79 & 93 \\
\hline & & Drinking & 124 & 0 & 35 & 1 & 0 & 0 \\
\hline & & Syrup bottle & 3 & 0 & 0 & 0 & 0 & $\dot{0}$ \\
\hline & & Water bottle & 0 & 0 & 0 & 0 & 0 & 0 \\
\hline & & Bleach bottle & 304 & 0 & 0 & 6 & 134 & 0 \\
\hline & & Food & 439 & 0 & 0 & 48 & 78 & 22 \\
\hline & & Baby bottle & 0 & 0 & 0 & 0 & 59 & 0 \\
\hline & Window & House & 0 & 0 & 0 & 74 & 0 & 0 \\
\hline & Jar & Cosmetic jar & 26 & 0 & 0 & 0 & 3 & 2 \\
\hline & & Food & 5 & 0 & 0 & 0 & 1 & 0 \\
\hline & & Unknown & 58 & 1 & 0 & 0 & 0 & 1 \\
\hline & Bowl & & 4 & 0 & 0 & 0 & 0 & 0 \\
\hline & Spectacles & & 2 & 0 & 0 & 0 & 3 & 0 \\
\hline & Lid & & 1 & 0 & 0 & 0 & 0 & 0 \\
\hline & Toy & Marble & 0 & 0 & 0 & 4 & 2 & 6 \\
\hline & & Unknown & 0 & 0 & 0 & 19 & 0 & 0 \\
\hline & Drinking & Tea & 33 & 0 & 0 & 4 & 5 & 0 \\
\hline & Cup & Drinking & 0 & 0 & 0 & 0 & 0 & 10 \\
\hline & & Unknown & 0 & 0 & 0 & 5 & 0 & 0 \\
\hline \multirow[t]{6}{*}{$\cdot$} & Plate & Food & 0 & 46 & 0 & 0 & 22 & 0 \\
\hline & Unknown & Mirror & 1 & 0 & 1 & 0 & 1 & 0 \\
\hline & & Button & 0 & 0 & 0 & 1 & 0 & 0 \\
\hline & & Bead & 0 & 0 & 0 & 0 & 2 & 0 \\
\hline & & Unknown & 2376 & 20 & 723 & 650 & 701 & 1192 \\
\hline & Totals & & 3784 & 67 & 901 & 17541 & 1100 & 1497 \\
\hline
\end{tabular}


Table 2. Guadalupe Ceramics by Structure.

\begin{tabular}{|c|c|c|c|c|c|c|c|c|}
\hline \multirow[b]{2}{*}{ Material } & \multirow[b]{2}{*}{ Sub-class } & \multirow[b]{2}{*}{ Object } & \multicolumn{2}{|c|}{ Russian } & \multicolumn{2}{|c|}{ Russian/Mexican } & \multicolumn{2}{|c|}{ Mexican } \\
\hline & & & $\begin{array}{l}\text { str. } \\
39\end{array}$ & $\begin{array}{l}\text { str. } \\
61\end{array}$ & $\begin{array}{l}\text { str. } \\
11\end{array}$ & $\begin{array}{l}\text { str. } \\
16\end{array}$ & $\begin{array}{l}\text { str. } \\
2\end{array}$ & $\begin{array}{l}\text { str. } \\
76\end{array}$ \\
\hline \multirow[t]{11}{*}{ Ceramics } & Earthenwar $\mathrm{E}$ & Bowl & 8 & 0 & 0 & 0 & 0 & 0 \\
\hline & & Plate & 5 & 0 & 21 & 0 & 2 & 0 \\
\hline & & Cup & 1 & 0 & 2 & 0 & 0 & 0 \\
\hline & & Tile & 2 & 0 & 0 & 1 & 0 & 0 \\
\hline & & Brick & 0 & 1 & 0 & 1 & 0 & 0 \\
\hline & & Pitcher & 0 & 0 & 0 & 1 & 0 & 0 \\
\hline & & Figurine & 0 & 0 & 0 & 0 & 0 & 0 \\
\hline & & Pot & 4 & 0 & 0 & 0 & 0 & 0 \\
\hline & & Crockery & 0 & 0 & 0 & 1 & 0 & 0 \\
\hline & & Door knob & 0 & 0 & 0 & 0 & 0 & 0 \\
\hline & & Unknown & 12 & 0 & 13 & 19 & 17 & 4 \\
\hline \multirow[t]{24}{*}{+} & Porcelain & Bowl & 33 & 0 & 0 & 7 & 0 & 1 \\
\hline & & Plate & 64 & 0 & 0 & 14 & 8 & 6 \\
\hline & & Cup & 19 & 0 & 0 & 0 & 0 & 0 \\
\hline & & Tile & 0 & 0 & 1 & 0 & 0 & 0 \\
\hline & & Brick & 0 & 0 & 0 & 0 & 0 & 0 \\
\hline & & Pitcher & 1 & 0 & 0 & 0 & 0 & 0 \\
\hline & & Figurine & 0 & 0 & 0 & 0 & 0 & 0 \\
\hline & & Pot & 9 & 0 & 0 & 0 & 0 & 0 \\
\hline & & Crockery & 0 & 0 & 0 & 0 & 2 & 0 \\
\hline & & Door knob & 0 & 0 & 0 & 1 & 1 & 0 \\
\hline & & Unknown & 106 & 0 & 22 & 94 & 154 & 55 \\
\hline & Plaster & Bowl & 0 & 0 & 0 & 0 & 0 & 0 \\
\hline & & Plate & 0 & 0 & 0 & 0 & 0 & 0 \\
\hline & & Cup & 0 & 0 & 0 & 0 & 0 & 0 \\
\hline & & Tile & 0 & 0 & 0 & 0 & 0 & 0 \\
\hline & & Brick & 0 & 0 & 0 & 0 & 0 & 0 \\
\hline & & Pitcher & 0 & 0 & 0 & 0 & 0 & 0 \\
\hline & & Figurine & 0 & 0 & 0 & 0 & 0 & 1 \\
\hline & & Pot & 0 & 0 & 0 & 0 & 0 & 0 \\
\hline & & Crockery & 0 & 0 & 0 & 0 & 0 & 0 \\
\hline & & Door knob & 0 & 0 & 0 & 0 & 0 & 0 \\
\hline & & Unknown & 0 & 0 & 1 & 0 & 5 & 0 \\
\hline & Tizon & Unknown & 2 & 0 & 1 & 1 & 0 & 4 \\
\hline & Totals & & 266 & 1 & 61 & 140 & 189 & 71 \\
\hline
\end{tabular}


Table 3. Guadalupe Ceramic Design Elements by Structure.

\begin{tabular}{|c|c|c|c|c|c|c|c|c|c|c|c|c|}
\hline Structure & Floral & Stripe & Emboss & Solid & Chinese & $\begin{array}{l}\text { Incised } \\
\text { earthen }\end{array}$ & $\begin{array}{l}\text { Emboss } \\
\text { earthen }\end{array}$ & $\begin{array}{l}\text { Polychr } \\
\text { earthen }\end{array}$ & Tiles & Scenic & Geo & $\begin{array}{l}\text { Sponge/ } \\
\text { Spackle }\end{array}$ \\
\hline \multicolumn{13}{|l|}{ STRUCT 76} \\
\hline Unit $1-0 \mathrm{~cm}$ & & & & & & & & & 1 & & & \\
\hline Unit $2.0 \mathrm{~cm}$ & 1 & & & 3 & & & & & & 1 & & \\
\hline $0-10 \mathrm{~cm}$ & 1 & & & 1 & & & & & & & & \\
\hline $10-20 \mathrm{~cm}$ & 1 & & & & & & & & & & & \\
\hline SURF COLL. & & & & & & & & & & & & \\
\hline IQA & & & & & & & & & & 10 & & \\
\hline $10 B$ & 1 & & 1 & 1 & 2 & & & & 1 & 2 & & \\
\hline $10 D$ & 1 & & & 4 & & & & & 1 & & & \\
\hline $11 \mathrm{D}$ & 1 & & & & 2 & & & & & 3 & & \\
\hline POSTHOLE & & & & & & & & & & & & \\
\hline 6 & 2 & & & & & & & & & & & \\
\hline TOTALS & 8 & 0 & 0 & 9 & 4 & 0 & 0 & 0 & 3 & 16 & 0 & 0 \\
\hline \multicolumn{13}{|l|}{ STRUCT 11} \\
\hline \multicolumn{13}{|l|}{ UNIT 1} \\
\hline $0-10 \mathrm{~cm}$ & 10 & & & & & & & & & & & \\
\hline \multicolumn{13}{|l|}{ UNIT 2} \\
\hline \multicolumn{13}{|l|}{$0-10 \mathrm{~cm}$} \\
\hline $10.20 \mathrm{~cm}$ & 9 & & & & & & 2 & 4 & 3 & 5 & & \\
\hline TOTALS & 19 & 0 & 0 & 0 & 0 & 0 & 2 & 4 & 3 & 5 & 0 & 0 \\
\hline
\end{tabular}




\begin{tabular}{|c|c|c|c|c|c|c|c|c|c|c|c|c|}
\hline Structure & Flocal & Stripe & Emboss & Solid & Chinese & $\begin{array}{l}\text { Incised } \\
\text { earthen }\end{array}$ & $\begin{array}{l}\text { Emboss } \\
\text { earthen }\end{array}$ & $\begin{array}{l}\text { Polychr } \\
\text { earthen }\end{array}$ & Tiles & Scenic & Geo & $\begin{array}{l}\text { Sponge/ } \\
\text { Spackle }\end{array}$ \\
\hline \multicolumn{13}{|l|}{ STRUCT 61} \\
\hline TOTALS & 0 & 0 & 0 & 0 & 0 & 0 & 0 & 0 & 0 & 0 & 0 & 0 \\
\hline \multicolumn{13}{|l|}{ STRUCT 39} \\
\hline \multicolumn{13}{|l|}{ UNIT 1} \\
\hline $0-10 \mathrm{~cm}$ & & & 2 & & & & & & & & & \\
\hline $10-20 \mathrm{~cm}$ & & & 1 & & 1 & & & & & & & \\
\hline $0-10 \mathrm{~cm}$ & 8 & & & 10 & & & & & & 2 & & \\
\hline $10-20 \mathrm{~cm}$ & 17 & & 1 & 6 & & & & & & & & \\
\hline $20-30 \mathrm{~cm}$ & 13 & 1 & & 9 & & & & & & & & \\
\hline $30-40 \mathrm{~cm}$ & 21 & 1 & & 16 & & & & & & & & \\
\hline $40-50 \mathrm{~cm}$ & 9 & 1 & & 11 & & & & & & 3 & & \\
\hline $50-60 \mathrm{~cm}$ & 4 & 1 & & 2 & & & & & & & & \\
\hline $60-70 \mathrm{~cm}$ & 6 & & & 2 & & & & & & & & \\
\hline $70-80 \mathrm{~cm}$ & 3 & & & & 1 & & & & & & & \\
\hline $140-150 \mathrm{~cm}$ & 1 & & & 1 & & & & & & & & \\
\hline TOTALS & 82 & 4 & 4 & 57 & 2 & 0 & 0 & 0 & 0 & 5 & 0 & 0 \\
\hline \multicolumn{13}{|l|}{ STRUCT 2} \\
\hline \multicolumn{13}{|l|}{ UNIT 1} \\
\hline $0-20 \mathrm{~cm}$ & 2 & & & & 11(Blue & & & & & & & \\
\hline \multicolumn{13}{|l|}{ UNIT 2} \\
\hline $0-20 \mathrm{~cm}$ & 1 & & 1 & & & & & & & & & \\
\hline \multicolumn{13}{|l|}{ UNIT 3} \\
\hline $0-20 \mathrm{~cm}$ & & & & 13 & & & & & & & & \\
\hline $20-40 \mathrm{~cm}$ & 2 & & & 1 & 4(Blue & & & 5 & & & & \\
\hline
\end{tabular}




\begin{tabular}{|c|c|c|c|c|c|c|c|c|c|c|c|c|}
\hline Structure & Floral & Stripe & Emboss & Solid & Chinese & $\begin{array}{l}\text { Incised } \\
\text { earthen }\end{array}$ & $\begin{array}{l}\text { Emboss } \\
\text { earthen }\end{array}$ & $\begin{array}{l}\text { Polychr } \\
\text { earthen }\end{array}$ & Tiles & Scenic & Geo & $\begin{array}{l}\text { Sponge/ } \\
\text { Spackle }\end{array}$ \\
\hline \multicolumn{13}{|l|}{ UNIT 4} \\
\hline $0-20 \mathrm{~cm}$ & 14 & 5 & & 17 & 4/4(Blue & & & 6 & & & 2 & \\
\hline $20-40 \mathrm{~cm}$ & 4 & & & 1 & & & & & & & & \\
\hline \multicolumn{13}{|l|}{ UNIT 5} \\
\hline $0-20 \mathrm{~cm}$ & 3 & 2 & 1 & & 1 & & & & & & & \\
\hline TOTALS & 26 & 7 & 2 & 32 & 24 & 0 & 0 & 11 & 0 & 0 & 2 & 0 \\
\hline \multicolumn{13}{|l|}{ STRUCT 16} \\
\hline \multicolumn{13}{|l|}{ UNIT 1} \\
\hline $10-20 \mathrm{~cm}$ & 1 & & & & & & & & & & & \\
\hline $40.50 \mathrm{~cm}$ & & & & & & & & & & & 1 & \\
\hline \multicolumn{13}{|l|}{ UNIT 2} \\
\hline $0-20 \mathrm{~cm}$ & 4 & & & & & & & & & & & \\
\hline $20-40 \mathrm{~cm}$ & 3 & & & 1 & & & & & & & & \\
\hline $40.60 \mathrm{~cm}$ & 2 & 1 & 1 & & & & & & & & & \\
\hline \multicolumn{13}{|l|}{ UNIT 3} \\
\hline $20-40 \mathrm{~cm}$ & 5 & 4 & 5 & 6 & & & & & & & 1 & \\
\hline \multicolumn{13}{|l|}{ UNIT 4} \\
\hline $40-50 \mathrm{~cm}$ & 2 & 1 & 1 & 1(Blue) & & & & & & & & \\
\hline \multicolumn{13}{|l|}{ UNTT 5} \\
\hline $0-20$ & 18 & 6 & 1 & 21 & 1(Blue) & & & 3 & & 2 & & 1 \\
\hline \multicolumn{13}{|l|}{ Posthole } \\
\hline$\$ 10 \mathrm{~B} 40$ & & & & & & & & & & 1 & & \\
\hline TOTALS & 35 & 12 & 7 & 29 & 1 & 0 & 0 & 3 & 0 & 3 & 2 & 1 \\
\hline STRUCT 17 & 0 & 0 & 0 & 0 & 0 & 0 & 0 & 0 & 0 & 0 & 0 & 0 \\
\hline
\end{tabular}


Table 4. Guadalupe Metal by Structure.

\begin{tabular}{|c|c|c|c|c|c|c|c|c|}
\hline \multirow[b]{2}{*}{ Material } & \multirow[b]{2}{*}{ Sub-class } & \multirow[b]{2}{*}{ Object } & \multicolumn{2}{|c|}{ Ruesien } & \multicolumn{2}{|c|}{ Rusxian/Mexican } & \multicolumn{2}{|c|}{ Mexican } \\
\hline & & & $\begin{array}{l}8 t r . \\
39\end{array}$ & $\begin{array}{l}\text { str. } \\
61\end{array}$ & $\begin{array}{l}\text { str. } \\
11\end{array}$ & $\begin{array}{l}\text { str. } \\
16\end{array}$ & $\begin{array}{c}\text { str. } \\
2\end{array}$ & $\begin{array}{l}\text { str. } \\
76\end{array}$ \\
\hline \multirow[t]{8}{*}{ Metal } & Tin & & 2230 & 1 & 0 & 90 & 21 & 104 \\
\hline & Iron & & 3421 & 47 & 3 & 2 & 221 & 391 \\
\hline & Copper & & 170 & 0 & 6 & 8 & 1 & 12 \\
\hline & Aluminum & & 58 & 152 & 36 & 15 & 1 & 12 \\
\hline & Steel & & 21482 & 7 & 854 & 217 & 6 & 14 \\
\hline & Graphite & & 7 & 0 & 2 & 0 & 0 & 3 \\
\hline & Brass & & 3 & 0 & 11 & 1 & 0 & 0 \\
\hline & Lead & & 0 & 0 & 2 & 0 & 0 & 0 \\
\hline \multirow[t]{3}{*}{-} & Silver & & 1 & 0 & 0 & 0 & 2 & 0 \\
\hline & Unknown & & 29066 & 41 & 322 & 2864 & 848 & 460 \\
\hline & Totals & & 56438 & 248 & 1236 & 3197 & 1100 & 996 \\
\hline
\end{tabular}

One cracked creamware pitcher (CRO349) located at $180 \mathrm{~cm}$. depth, had a Czechoslovakian maker's mark which neither Ensenada nor San Diego historians had ever seen before. Dr. Glenn J. Farris of the California Department of Parks and Recreation noted that the mark appears to be from $\mathrm{H}$. Wehinger and $\mathrm{Co}_{\mathrm{o}}$, in Bohemia, a company which made household porcelain for Eastem Europe between the years of 1905 and 1945 (Rontgen, 1981:248,420). H. Wehinger and Co. was an earthenware factory, which had been in operation since 1817, when George Friedrich Hom founded it, but from 1822 to 1905 , under the J \& B Boyer name of the "Bros. Horn" had produced dinnerware, plus decorative and household earthenware. Between 1905 and 1918, the company's maker's mark had been a squat vessel (with or without top) with a prominent " $W$ " centered in it. Bohemia, now Czechoslovakia, was a central region, which was crossed by many Molokans traveling from the Black Sea to ports of emigration in Northem Germany. It was hypothesized that a Molokan woman sometimes purchased a small piece such as this and carried it with her to the colony, where the evidence was later found, indicating that the name Czechoslovakia dates only from the establishment of the modern republic in 1918. 
Table 5. Guadalupe Metal Artifacts by Structure.

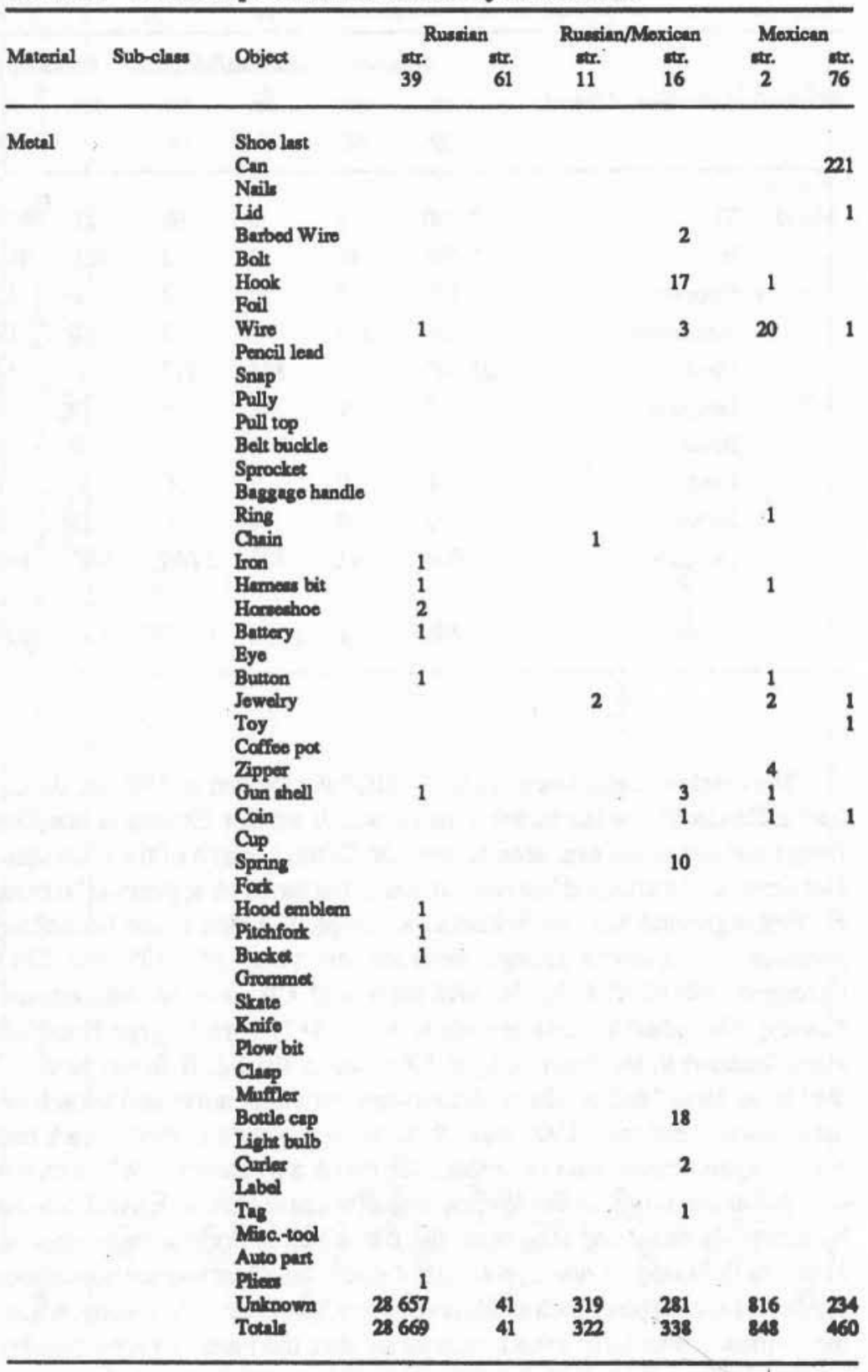


Table 6. Guadalupe Bone by Structure.

\begin{tabular}{|c|c|c|c|c|c|c|c|c|}
\hline \multirow[b]{2}{*}{ Material } & \multirow[b]{2}{*}{ Sub-class } & \multirow[b]{2}{*}{ Object } & \multicolumn{2}{|c|}{ Russian } & \multicolumn{2}{|c|}{ Russian/Mexican } & \multicolumn{2}{|c|}{ Mexican } \\
\hline & & & $\begin{array}{l}\text { str. } \\
39\end{array}$ & $\begin{array}{l}\text { str. } \\
61\end{array}$ & $\begin{array}{l}\text { str. } \\
11\end{array}$ & $\begin{array}{l}\text { str. } \\
16\end{array}$ & $\begin{array}{c}\text { str. } \\
2\end{array}$ & $\begin{array}{l}\text { str. } \\
76\end{array}$ \\
\hline \multirow[t]{8}{*}{ Bone } & Animal & Cow & 2 & 0 & 1 & 2 & 0 & 3 \\
\hline & & Pig & 0 & 0 & 0 & 5 & 0 & 0 \\
\hline & & Chicken & 0 & 0 & 0 & 0 & 0 & 0 \\
\hline & & Deer & 0 & 0 & 0 & 0 & 0 & 1 \\
\hline & & Rabbit & 1 & 0 & 0 & 1 & 0 & 0 \\
\hline & & Bird & 0 & 0 & 0 & 1 & 0 & 0 \\
\hline & & Unknown & 56 & 1 & 11 & 79 & 20 & 18 \\
\hline & Totals & & 59 & 1 & 12 & 88 & 20 & 22 \\
\hline
\end{tabular}

True polychrome types of earthenware (terracottas) of local Mexican make were not present. Cheap, lead-glazed, types of red earthenware (like Tecate redware) were available in both the Ensenada and Tijuana markets, but Susana seems to have selected inexpensive whiteware for her domestic use. She had the remains of a plain redware vessel which fitted back into two small pots. For cataloguing purposes (since so many different archaeologists were called in to deal with the bulk cataloguing), the terracottas were sorted from the stoneware; from the whiteware/ironstone and from the porcelains; and were then sorted a second time for subtype, and a third time for design elements. The terracottas were of a crudely-prepared paste with grit temper, poor firing technique, and of local manufacture. These were difficult to date, as they are made with crude slips and burnishes which were made from the 1850 's up to the present. They are still being sold to tourists in towns all along the U.S.-Mexican border. Two pieces of Tizon brownware, the Kumeyaay Indian native ware, were found, attesting to the Kumeyaays' long occupation of the valley prior to Russian contact.

Table 4 illustrates the difficulty of sorting the dump at Structure 39 by metal type. Fortunately, a crew member was a metallurgical engineer, and took charge of the metals identification through a variety of techniques (e.g. lifting with magnets). Tin was found in the form of badly-fragmented tin-plated cans. Steel was primarily truck parts. Table 5 illustrates artifact types, the bulk of the Kachiriski metal artifacts being unidentified. 
Table 7. Guadalupe Seeds by Structure.

\begin{tabular}{llrrrrrr}
\hline & \multicolumn{3}{c}{ Russian } & Russian/Mexican & \multicolumn{2}{c}{ Mexican } \\
Material & str. & str. & str. & str. & str. & str. \\
& 39 & 61 & 11 & 2 & 16 & 76 \\
\hline \multirow{3}{*}{ Seeds } & & & & & & & \\
& & 0 & 2 & 3 & 0 & 0 & 13 \\
& Peach & 0 & 0 & 4 & 0 & 0 & 0 \\
& Apricot & 0 & 1 & 3 & 0 & 0 & 0 \\
Peanut & 2 & 0 & 0 & 2 & 1 & 0 \\
Olive & 0 & 0 & 0 & 0 & 0 & 1 \\
Pomegranate & 0 & 0 & 0 & 0 & 2 & 0 \\
Corn & 54 & 0 & 8 & 1 & 4 & 0 \\
Unknown & 56 & 3 & 18 & 3 & 7 & 14 \\
Totals & & & & & & & \\
\end{tabular}

Animal bone (Table 6) was uninformative, as Dr. Lynn Christenson, a zooarchaeologist at San Diego State University, could not determine species for the majority of the items. Animal bone was so poorly preserved throughout the colony that its only use is as part of a tally of the presence or absence of species considered unclean by the Molokans. Susana had no such species. Like animal bone, seeds were few, and primarily unidentifiable.

Susana had plastic combs, plastic medicine droppers, a phonograph record, and what was a small amount of miscellaneous plastic for the large numbers of artifacts found in Unit 2. This would be in keeping with her poverty, and professed lack of interest in modern innovation (Table 8).

The total picture, therefore, from the late 1940's to the early 1950 's, is of a somewhat acculturated (Russian tea glasses, but Victrola phonograph records) older female living with those of her juvenile children who were as yet too young to emigrate to the United States. Her source of income (laundress) is indicated in the large number of glass bleach containers and food bottles. The number of bottles of alcoholic beverages remains a puzzle, as everyone interviewed said there was no alcohol use. It may be that family visited from Los Angeles on occasion, and contributed to the numbers, or that the bottles were being reused by Susana, not an uncommon occurrence in rural areas. 
Table 8. Guadalupe Plastics by Structure.

\begin{tabular}{llrrrrrr}
\hline & & \multicolumn{7}{c}{ Russian } & \multicolumn{2}{c}{ Russian/Mexican } & \multicolumn{2}{r}{ Mexican } \\
Material Sub-class & Object & str. & str. & str. & str. & str. & str. \\
& & 39 & 61 & 11 & 16 & 2 & 76 \\
\hline \multirow{3}{*}{ Plastics } & Fishing line & 1 & 1 & 0 & 0 & 0 & 0 \\
& Toy & 2 & 3 & 0 & 0 & 4 & 0 \\
& Pot & 0 & 0 & 0 & 0 & 0 & 43 \\
& Comb & 2 & 0 & 0 & 2 & 2 & 0 \\
& Cap & 3 & 2 & 0 & 1 & 0 & 2 \\
& Eyedropper & 1 & 0 & 0 & 0 & 0 & 0 \\
& Record & 1 & 0 & 0 & 26 & 0 & 0 \\
& Button & 1 & 0 & 1 & 12 & 1 & 1 \\
& Toothbrush & 0 & 0 & 0 & 14 & 0 & 0 \\
& Flashlight & 0 & 0 & 0 & 9 & 0 & 0 \\
& Utensils & 2 & 0 & 0 & 0 & 0 & 0 \\
& Unknown & 41 & 3 & 27 & 27 & 18 & 205 \\
& & 54 & 9 & 28 & 91 & 25 & 251 \\
\hline \multirow{2}{*}{ Totals } & & & & & & &
\end{tabular}

\section{Structure 61. Andrés Moiseich Samaduroff}

Two of the elderly sons of Moises Samaduroff and Tanya Klistoff were still living in Guadalupe at the beginning of this study in 1983. David Samaduroff had married a Mexican woman, a local political party activist; but his brother Andrés (standard Russian Andrei Moiseevich Samadurov) had married Petra Pavloff, a "pure Russian" woman. He and Petra had several children, of whom teenagers Andrés and Tanya were still at home, and daughter Surkia returned from a job in Mexicali on a regular basis (Figure 3, Structure 61 and Figure 5). The children had bright "carrot-red" hair and blue eyes, but spoke only Spanish to us. They lived on their grandparents' parcel of land. The Samaduroffs were the last family of rusos puros left in Guadalupe, which at one time had numbered more than 100 families, and hundreds of members. All the Samaduroff children had been baptized Molokan, and maintained the religious affiliation. Teenager Andrés was a special friend, who provided us with photographs from a private collection of mementos that he kept. He seemed to feel that the 
colony was still special. He was torn between how to maintain the old ways, which he valued, and yet deal with new ways, as he searched for a reason to emigrate to "the other side". He maintained the Russian cemetery beautifully, showing up on countless Sundays to rake and cut weeds, clean headboards and maintain fences.

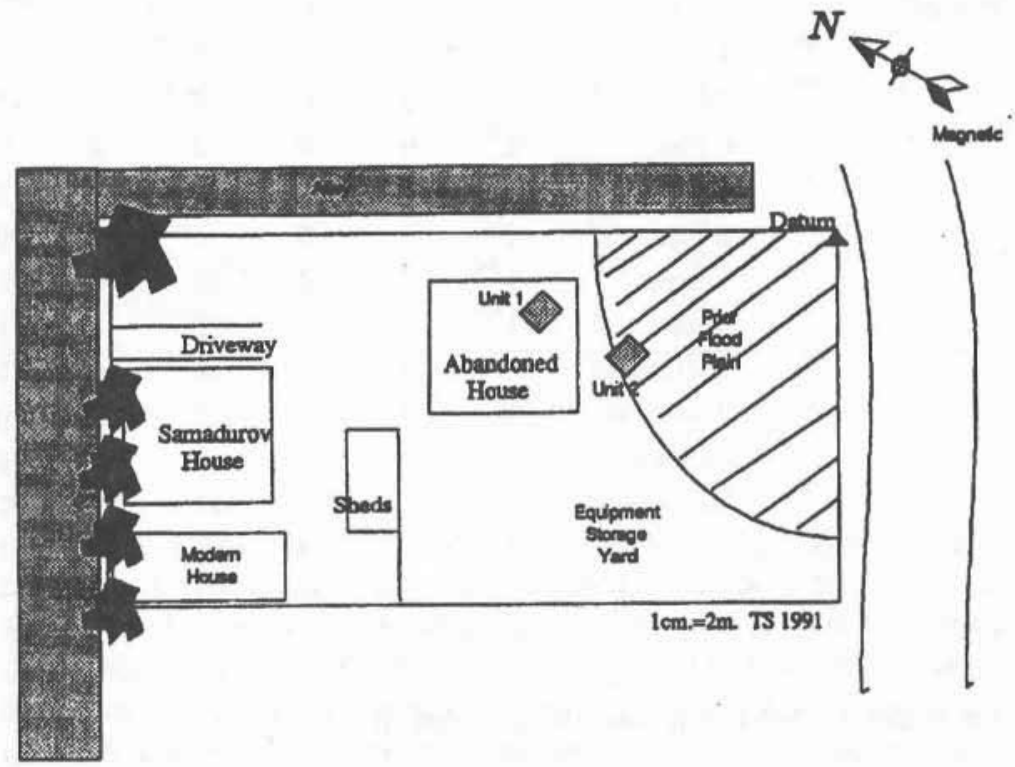

Figure 5. Plan of Andrés Moiseich Samaduroff's House.

Artifacts from the Samaduroff house were limited in number. Two excavation units were put in on October 6,1985. Unit 1 was placed in an abandoned structure behind the main house, and was cut to $70 \mathrm{~cm}$. Unit 2 was an abandoned latrine and went to only $40 \mathrm{~cm}$. before flood damage erased it. Unfortunately, only 336 artifacts came from the Samaduroff house. Flood damage mixed a few older bottles with modern plastic, creating a unit of secondary deposition with no integrity. Unit 1 produced an aluminum dump of badly-corroded tin-plated sanitary cans (as opposed to the hole-and-cap, or solder-drop cans [Busch, 1981]), as well as iron tractor parts. House 61 , as interesting as its acculturated occupants were, yielded few artifacts that would be of use here. 


\section{Structure 11. Moisés Grigorich Samarin}

Moisés Grigorich Samarin's (standard Russian Moisei Grigorovich Samarin) house (Figure 3, Structure 11, Figure 6) is located toward the southern end of the line-village at the connection with Highway 3 . His house was built for his parents Grigori Samarin and Masha Pivovarov by his grandparents Timofei and Tanya Samarin. (The grandparents came from Russia, and lived in what was the original 1834 mission building at the colony [Figure 3, Structure 47]). Moisés Samarin (who was alive at the time of the excavation but is now deceased) married a Mexican woman, someone considered by Molokans as ne nash or "not one of ours". His lovely wife was Lola Lopez de Samarin, and they had four children: Andrés, Rosa, Eloisa and Antonio, most of whom were married and on their own. Rosa had married a half-Russian, half- Mexican man from the Kachiriski family, making their offspring still half-Russian, half-Mexican. Moisés' family was very social, and very much at home in two worlds.

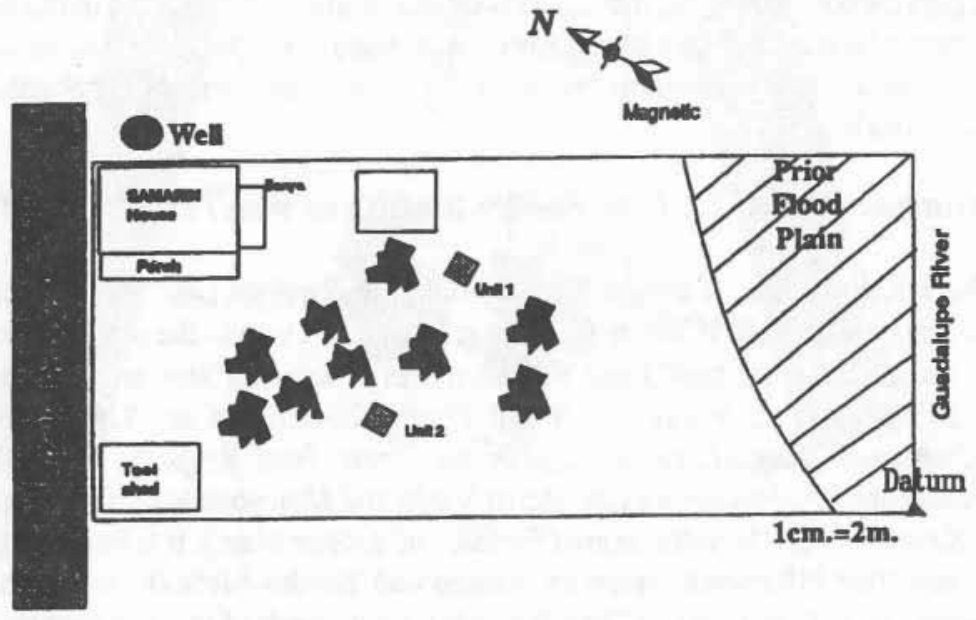

Figure 6. Plan of Moisés Grigorich Samarin's House. 
Excavations took place on October 5th and 6th of 1985. Two (1 1 1 meter) units were placed, based on surface concentrations of artifacts. Unit 1 was excavated to a depth of $40 \mathrm{~cm}$, and Unit 2 to a depth of $50 \mathrm{~cm}$. Both were kitchen middens dating from the late 1940's to the present, with recent bone refuse being scattered on the surface. The ceramics were noteworthy, in that types of polychrome earthenware were selected over porcelains. Colorful types of redware (e.g. CRO-1363) in polychromes of olive green and yellow on a deep red paste were noted. Polychrome serving vessels were noted at the time of ethnographic interview in use in the kitchen and outside serving areas. The ceramic collection was primarily of locally-produced polychromes painted in bright combinations of color, instead of the ironstone/whiteware, with perhaps a small floral motif of Structure 39, the Kachiriski house. Floral motifs recorded in Table 3 are misleading, as the flower design at the Samarin's was usually hand painted under glaze, the flowerettes being more than $5 \mathrm{~cm}$ in diameter, and not the transfer-print motifs of small pastels that Susana selected at Structure 39.

The Samarin household trash reflected the ethnographic data fairly well. The presence of a Mexican wife was notable in a different selection of polychrome ceramics, sources of food materials, different trash disposal patterns (scattered in small dumps over the entire property, and in sheet trash, as opposed to a centralized dump area used year after year by Susana Kachiriski).

\section{Structures 16 and 17. Juan Vasilich Rogoff and Ivan Pavlich Rogoff}

The house of Juan Vasilich Rogoff (standard Russian Ivan Vasilievich Rogov) was located in the middle of the colony at a turn in the road known as La Vuelta ("the turn") and in Russian as et konets ("this end" of the street) (Figure 3, Structure 16 and Figure 7). Known as "Chino" or "Chinaman" Rogoff, to distinguish him from Juan Rogoffs, of other Guadalupe families, he was the son of Vasili and Matryona Rogoff (Figure 3, Structure 16), He had a brother Basilio and a sister María. It is interesting to note that informants (such as George and Basilio Mohoff) called the father "Vasili" and the son "Basilio", a linguistic mark of the acculturation taking place. Chino's first wife was a Nadia Rogoff, and they had an all-Russian daughter, Vera. His second wife was ne nash and they had a son, Juanito.

Excavations took place in Structure 16 on October 20,21 and 22 of 1990. Five (1 1 1 meter) units were dug, including two units in the basement of the abandoned house. Room functions were identified (by Russian informants like Pedro Kachiriski, who still lives in the valley) as sala, 


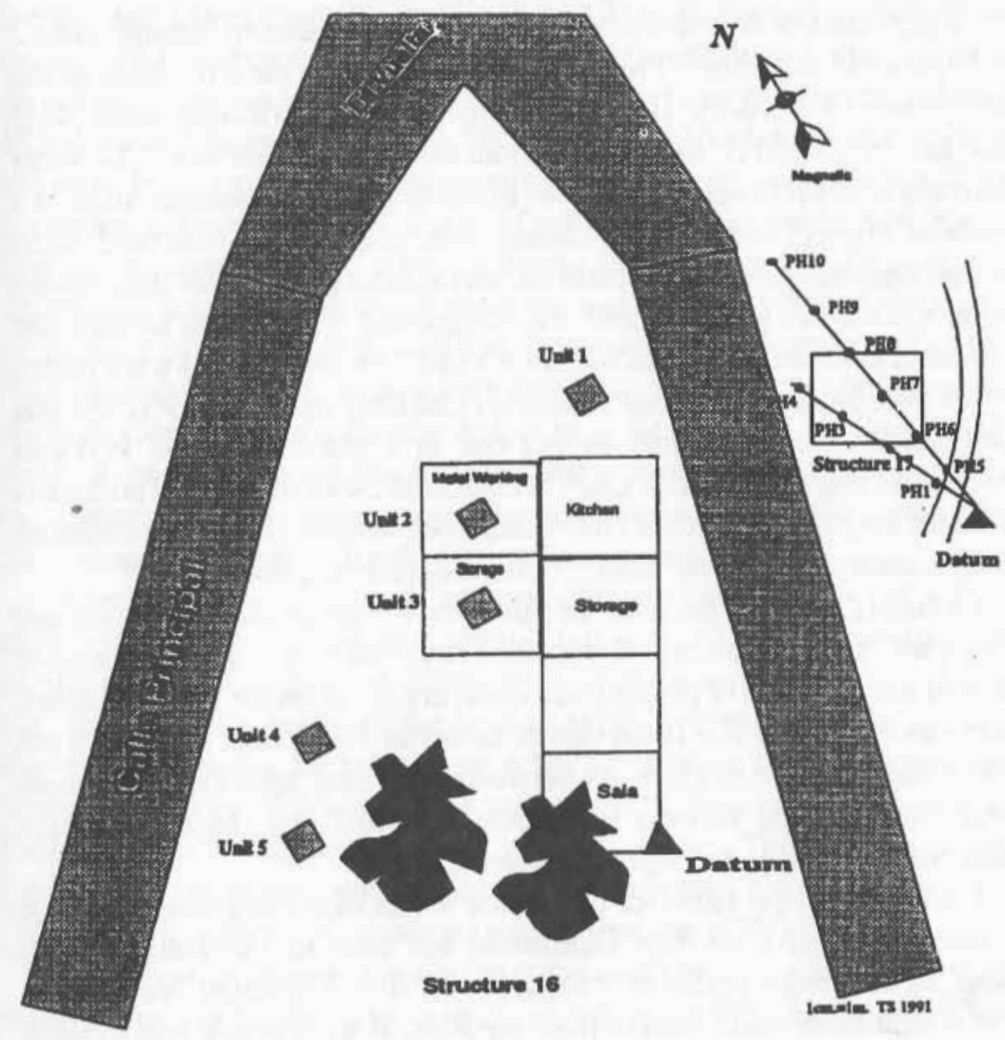

Figure 7. Plan of Juan Vasilich Rogoff's House. 
kitchen, storage areas, and a metal shop. Artifacts included some objects pertaining to males, others to females: such as snuff boxes, or nail polish bottles and metal hair curlers. Children's toys were in evidence. Glassware (Table 1) included beer and liquor bottles. Four fragments of Russian tea glasses were located in good primary deposition.

A pig's mandible and four teeth were located scattered through units 2 and 3, the old basement. The basement was an area used for trash in the later years of the colony, since burning or hauling it to a centralized place was too much effort for the demoralized colony numbers. The clear association of undeniable pig bones at depth in these basement areas is a remarkable tag of the degree of change undergone in this household, since the ban against intermarriage, and the ban against eating other than kosher foods was the last to be ignored. Two fragments of LP record albums and a Victrola label showing "His Master's Voice" (from the U.S.) were found, the Victrola being a noted sign of acculturated families (Story, 1960:38), and even Susana Kachiriski had an old one in a place of honor. Fourteen tooth-brush fragments were located in association with two human premolars extracted long after caries had hit the inner root. Mohoff (1992a:53) discusses home dentistry and pulling teeth by tying the tooth to a doorknob.

Ceramics were dominated in numbers by porcelain and ironstone/ whiteware, but the presence of colorful Tecate redware is noted. Examples showed the presence of polychrome earthenware in bright blues, yellows, greens and oranges. The floral design tallies in Table 3 once again do not reflect the dramatic differences between the floral tableware used here (large, handpainted flowers in random patterns) and those of Susana Kachiriski's small transfer-print flowerettes.

Interestingly, the presence of a black basalt mano fragment of a type of stone not native to Baja California, but used in Mexican kitchens primarily for the preparation of masa for tortillas was noted. No Russian wife would have made corn or flour tortillas, as their staple was a raised black wheat bread made in an outdoor oven or pechka. The mano indicates a dietary change for Chino Rogoff, in keeping with the meals made by a Mexican wife. Even though seeds were scarce through all the sites, the only corn husks or seeds found were those of Chino's household (Table 7). A goose skeleton was found in the basement area, the Russians being the first to breed geese in Baja Califomia, and geese being a characteristic Russian food, for which Rogoff may have maintained a fondness.

Structure 17 was across a side street from Structure 16, and had belonged to Ivan Pavlich Rogoff (standard Russian Ivan Pavlovich Rogov). A posthole series found no artifacts and no further analyses were made. 


\section{Structure 2. Grigori Danielich Afonin}

Grigori Danielich Afonin's (standard Russian Grigori Danielovich Afonin) house was at the end of the colony away from the poblado at Highway 3 (Figure 3, Structure 2, Figure 8). At later dates, houses such as Basilio and Maria Rogoff's (not on Figure 3) were built even farther out on the road to escape serious flooding, but for all intents and purposes Structure 2 was the end of the original colony. Grigori's parents, Daniel Mitrafonich Afonin and Matrona Tolmasoff, first lived in Structure 5 near the river, but flooding forced the family up and back to Structure 2 in 1910 , according to George Mohoff. Grigori Danielich Afonin and his wife Katya had ten children there and inherited all the father's property. Grigori was a man with innovative ideas; among those were, in the 1920's, the planting of 50 acres of grapes and the drilling of artesian wells. In the 1940's Mrs. Afonin sold the house to Mexican nationals, a couple by the name of Romulo Cota and Doña Pancha de Cerda. A Sr. Ferro of the Bodegas de Santo Tomas purchased the property subsequently, and at the time of excavation the house was occupied by an older Mexican male tenant who lived alone, and worked for the various grape growers.

Structure 2 was excavated September 30 and October 1 of 1989. Five units were placed, and varied in depth from 40 to $100 \mathrm{~cm}$. The units were finally dated as being of the same time period: from 1950 to 1960 . I was uncertain about the nature of the deposits at first, and in particular, whether they were Russian or Mexican in ethnicity. Suddenly in Unit 4, an elaborate brass medallion of Nuestra Señora de Guadalupe, the patroness of Catholic Mexico (Ewing, 1949), was recovered. The corrosion was marked, but the woman with the crescent moon under her feet surrounded with seven imitation red stones was quite apparent (CRO-1236).

Infant bottles appeared, a demographic marker, as infants were more common in this family than in those of the aging Russian householders whose homes have been reported so far. Tea glasses in the Mexican family's trash were a puzzle until depths were checked, and all were found to lie between 0 to $10 \mathrm{~cm}$, in depth. The entire Afonin yard had been shallowly ploughed at one time or another, and furrows were in evidence. This allowed for some artifacts of Russian usage to be dragged along over the entire surface of the property, contaminating upper levels of the Mexican trash pits.

There was gathered a mirror fragment which was one of the items that I hypothesized, in advance of the ethnographic research, to be a vanity item, probably not in keeping with Molokan mores. Finding this apparent sign of acculturation, I was interested to see it appearing also at the homes 


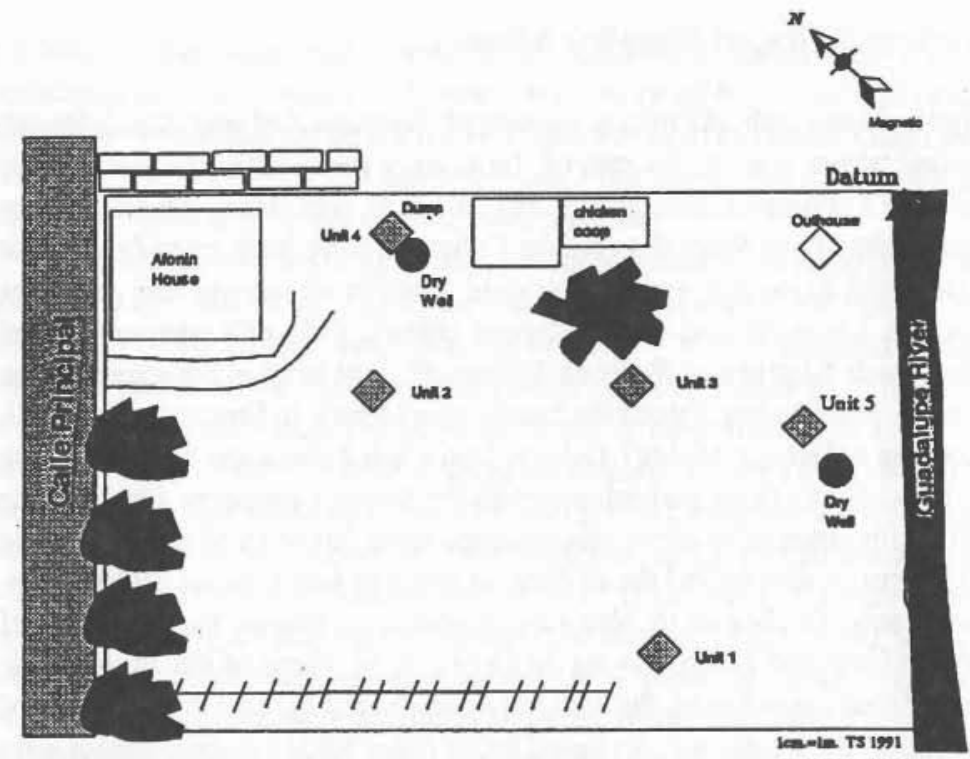

Figure 8. Plan of Grigori Danielich Afonin's House.

of Susana Kachiriski and Moisés Samarin. Statistically insignificant in small numbers, it nevertheless appeared. Upon closer ethnographic checks, however, it became apparent that the Molokans used mirrors to call the workers in from the fields when lunch or supper was ready, and that they also had a completely utilitarian usage having nothing to do with vanity.

Ceramics were brightly designed, hand-painted florals and solids. Floral design elements (Table 3) were large and nonsystematic in their distribution around the vessels. The presence of the largest number of "Blue Willow" fragments (19) in this highly-acculturated household contrasts with the avoidance of the Chinese dragon and pagoda themes by the traditional Molokans.

Two fragments of silverplate were located in the refuse, plus an inexpensive costume-jewelry heart locket. An interesting reuse of coat hangers, prominently seen on the walls of the Mexican residences in the valley, but never seen on the walls of Russian homes, was the twisting of the cut ends around the bent loop, which made all-purpose hangers. It supported the idea that the "reuse" of refuse was dramatically different between Molokan and Mexican families in selection of items as well as 
the prevalence of the reuse. The total picture of the Afonin house (which should probably be called the Cota house) was of a Mexican households with more traditional values than those of the half- Russian, half-Mexican household (Samarin or Chino Rogof) viewed to date.

\section{The Samarin Almacen}

Figure 9 illustrate the almacen, or warehouse, built by Grigori Timofeovich Samarin, who had many horses and needed corrals for them, according to family members. The almacen was always a storage area stocked with bales of hay. This was the first property test excavated on September 28 and 29, and October 5 and 6 of 1985, because the current property owners Sr. and Sra. Héctor Fuentes agreed to let us work there. Undocumented at that time, it seemed a good beginning point for allowing the Russian and Mexican villagers to get to know us and follow us around. It brought on some initial suspicion, as they thought we were local government surveyors with transit equipment. After a designated crew member passed each bag of artifacts around for a while, they became convinced we really were looking for basura or "trash".

The two units excavated (Figure 9), and the posthole series at the almacen, showed little subsurface material. I collected all the modern surface material by quadrants as a documentary of the village as it is today. The almacen now sits near small houses of Mexican workers and their families, and a small convenience store labeled Licores. Primarily scattered trash from contemporary households, it was the final sample needed to compare the living quarters of rusos puros, rusos-mexicanos and mexicanos excavated later.

Almacen trash was primarily machine-made beer and liquor bottles, which were larger in size than for the other houses studied so far, because they had been recently discarded. Only four Mexican earthenware pieces and 55 shards of whiteware were located, but when these were pieced together they were found to be pottery curios, and not the utilitarian ware of Susana Kachiriski, for example. Four Late Prehistoric to Early Historic Tizon brownware shards were collected, merely an indicator of the continuous occupation of the valley from prehistoric times to the present, and the problem of the intermixing of components in surface collections.

Iron car parts, tin and aluminum cans tossed from the side of the road, as well as from the small homesteads behind the convenience store, were collected. Large numbers of plastic forks and knives, broken flower pots, Japanese-made "flip-flop" sandals, etc. showed the high usage of plastics over a period of time. Food products showed a definite predilection for 
products with brand names such as Bufalo, Barrilitos and Salsa Brava. Although former Molokan colonists, such as Mohoff (1992a), remember eating jalapeño chiles with delight, the basic Russian diet was based upon geese, chickens, noodles and wheat bread with honey. Even after villagers stopped following kosher rules, they maintained some traditional tastes, according to Lola Lopez de Samarin and other informants.

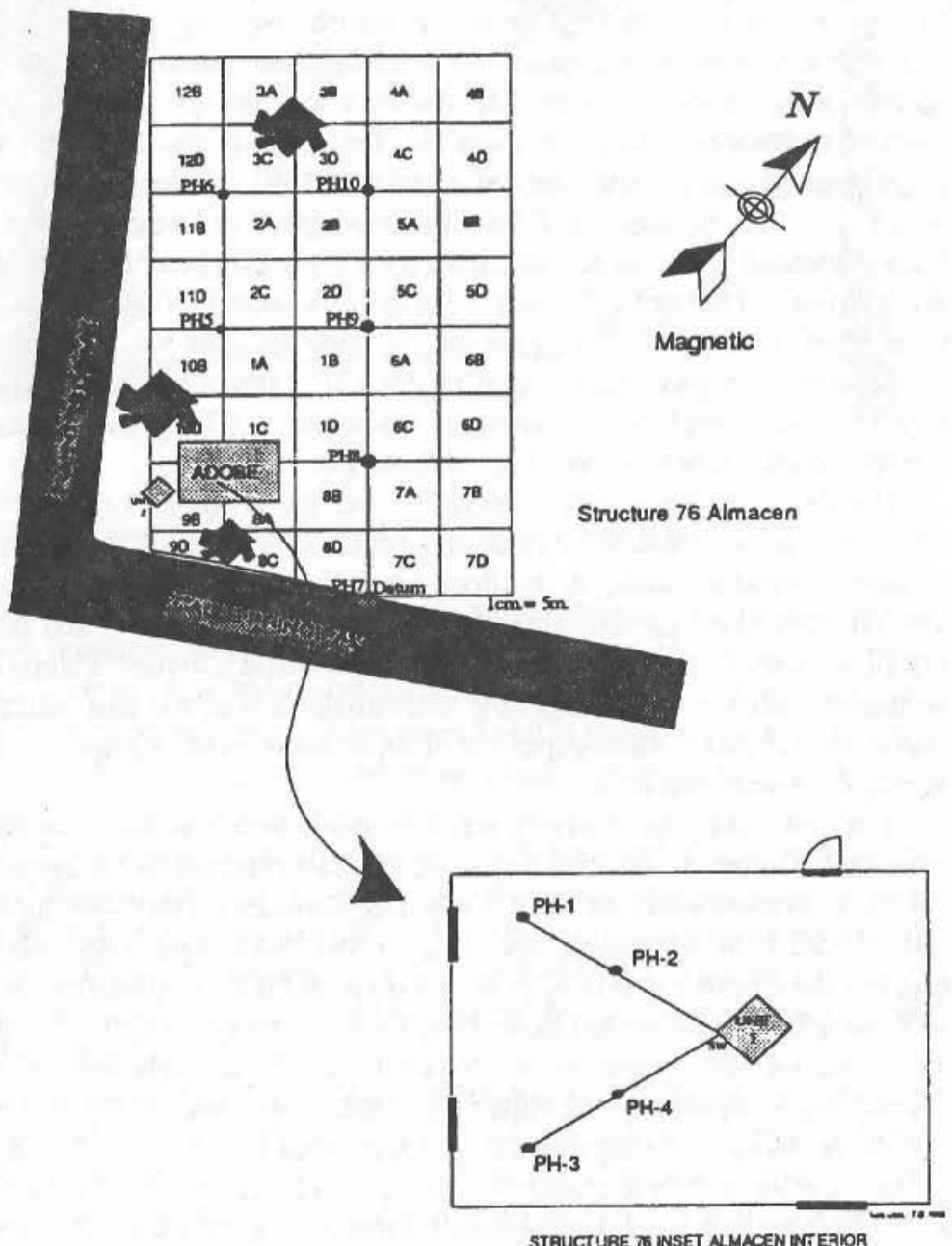

Figure 9. Plan of Samarin's Almacen. 


\section{CONCLUSIONS}

The Guadalupe Valley project actually began in the 1970 's when I was a student of East European prehistory. Interested in the origins of Indo-European languages, I could not detect the ethnic differences my colleagues attributed to prehistoric migrations in the archaeological record of Eastem Europe in 4,000 B.C. On the one hand, V. Gordon Childe noted (1942:17) that the subtle cultural differences among people as closely related as the British and the U.S. Americans of New England, would show up in the table manners and different shapes of forks, knives and spoons. Determining ethnicity by the objects people owned was possible, and worthy of attention. On the other hand, followers of Julian Steward (1955:82) were citing examples of ethnically-related peoples whose material cultural inventories were totally different, while ethnically different peoples could sometimes be found to own remarkably similar things. They seemed to be saying that the search for a correlation between material culture and ethnicity was fraught with difficulty. How could we address these potential problems? Just how accurately could I, as a prehistoric archaeologist, identify ethnic origin? If he or she worked with collections from sites where the settlers were still alive to critique the analyses with accuracy, could I learn techniques to clarify the identification of small and large-scale migrations?

Determined to try, and with permission from INAH in Mexico City, my crew and I placed test excavations at seven bouses of peoples of different ethnic backgrounds, and catalogued and analyzed their artifacts for evidence of points of ethnic origin. Ethnicity seemed to be an issue everywhere we went in this rural Baja California community. People classified me as the prahoslavnaya (ne nash), or "Orthodox" ("no one of us") or laughingly as la americana loca who was looking for old garbage. Our best success at obtaining farmers' permission to excavate, was when my Japanese-American spouse met the Japanese-Mexican owner of a formerly Russian homestead, and we were allowed access to all his property forevermore; thus I, Irish by descent, became la japonesa. I was looking for the material cultural evidence of ethnicity, the dymanics of which we were experiencing during our residence in the Guadalupe Valley.

Our relative success in correlating material culture with ethnicity was dramatic. With the introduction of the evidence of written language (as noted on cemetery headstones or household mailboxes), not only could we pinpoint East European origins for the population, but also the fact that they were sectarians (but not necessarily Molokans). Excavation weekends sometimes involved testing households for which there was no 
ethnographic background at the time of arrival on the site. This provided us with a chance to maintain different hypotheses as to whose bomestead it was, and the nature of the ethnic categories of the former occupants.

Without the evidence of writing and the religious messages characteristic of fundamental Christian sectarians, the evidence was still strong. As if we had been dropped into Russia (Dmitrieva, 1982-1983; Gerhart, 1974), all one had to do was open one's eyes to see the differences in the Guadalupe colony: the settlement pattern of the street-village, the roofs gabled to "keep off the snow" in a Mediterranean climate, the burial of the dead six feet down and two feet over into the side of the grave pit to "keep out the wolves". The unusual diet of geese, as well as the avoidance of non-kosher foods, was noteworthy, especially in regard to the techniques of butchery. The avoidance of salsa and spicy condiments, and the absence of tools for the preparation of tortillas showed a deviation from the typical Baja California diet. The material culture of samovars, carved wooden master serving spoons, plain or plainly-decorated ceramics, non-decorative buttons, and little or no jewelry, was observed. Ethnographically, the physical appearance (bright red hair and blue eyes) of the Samaduroff children in old photographs was evidence of genetic variability in the valley.

Does material culture correlate with ethnicity? It was represented in the Guadalupe Valley excavations, not as a statistical phenomenon, but as a difference in the presence or absence of characteristic items. For example, no pork remains were ever noted in purely Molokan bousehold trash pits, and no Mexican cookware such as metates, manos or morteros. Ceramic design elements were the strongest single piece of information in the detection of ethnicity. If kitchen middens could be located (in the Mexican households it was sheet trash, in the Molokan households it was trash dumped away from the structure and towards the river), the selection of porcelains versus earthenware, and the ceramic design elements (pastel Victorian florals versus multiple-colored florals with wide petals) were easy. Food jars of various makes were the next most useful determinant of ethnicity, and in particular, the salsas. The presence or absence of liquor bottles was not useful, because the colony had become acculturated from its earlier probibition of liquor, the avoidance of which was formerly a characteristic of Molokan faith.

\section{Archaeology and Ethnicity}

Does material culture therefore reflect ethnicity? The material culture of the Guadalupe Valley, as reflected in the test excavations here, confirms 
the presence of a group of Russian sectarians in northern Baja California. Strong local patterns characteristic of the Spanish (1533 to 1769, and 1769 to 1821) and Mexican (1821 to present) periods include large ranchos and small rancherias spread miles apart; houses of adobe bricks and red tile roofs; a dietary complex of com and beans; and native vegetation and fauna, plus a highly-symbolic Roman Catholic religious background. With this local pattem appears a second one, a departure within the Guadalupe Valley that begins in 1905, and has roots in Russia. Both indigenous and "intrusive" populations have left their marks.

If what I hypothesized at the time of excavations confirms what $I$ have since tracked ethnographically, I could have done it without the historic circumstances of supporting informants, personal letters, documents and historical archives. The Guadalupe Valley study is just too fresh, too contrastive in material plans for living, for a student of technology to miss. The Native American pattern (San Dieguito, Milling Stone, Late Prehistoric or Yuman) and the historic periods described above leave characteristic traces in the stratigraphy. The twentieth century Guadalupe Valley material is dramatic in its differences.

\section{The Saving of Molokan Russian Heritage}

The death of any Utopian settlement brings sadness to those who remember the idealism and hope with which it was founded. In particular, members of the Guadalupe colony (in which a "piece of heaven" was transplanted many miles at great cost), were grieved to have it disappear after only 53 years. This study documents the coming, colonization and disappearance of Russian immigrants to Baja California. The project publicized or even stimulated more Molokan and Mexican research into the topic (e.g. Lisizin, 1984; Mohoff, 1992, and the as-yet-unpublished work of Alfredo Gomez Estrada of the Museo Regional of Mexicali, Baja California) A particularly valuable piece of ethnographic data, the historical base map of Figure 3, was produced by George Mohoff at the suggestion of Katherine Abakumoff and myself. Furthermore, the governor of Baja California dedicated in perpetuity the Museo Regional de Guadalupe, an original Molokan Russian farm house which had been converted to display photo murals and artifacts from the "Saddles and Samovars" exhibit done on the Molokans for the San Diego Museum of Man in 1988. At least a little of the heritage of the Molokans (su patrocinio) has been saved, and an ongoing, published database has been provided for the general public, for science and for the Molokans themselves. 


\section{BIBLIOGRAPHY}

BARCLAY, Harold B. 1967. "Plain and Peculiar People". Alberta Anthropologist 1(3):4-14.

BARTH, Frederick, 1969. Ethnic Groups and Boundaries. Boston: Little, Brown.

BUSCH, Jane. 1981, "An Introduction to the Tin Can". Historical Archaeology, 15(1):95-10.

CHILDE, V. Gordon, 1942. What Happened in History. New York: Penguin.

DMITRIEVA, S.I. 1982. "Architectural \& Decorative Features of the 1983 Traditional Dwelling of the 1983 Mezen' River Russians". Soviet Anthropology XXI(3):29-54.

EWING, J. Franklin. 1949. "The Religious Medals". In Franciscan Awatovi: The Excavation and Conjectural Reconstruction of a 17th Century Spanish Mission Establishment at a Hopi Indian Town in Northeast Arizona, edited by Ross Gordon Montgomery, Watson Smith and John Otis Brew, Papers of the Peabody Museum of American Archaeology and Ethnology, Vol. XxxvI, Reports of the Awatovi Expedition, No.3. Cambridge: Peabody Museum, Harvard University.

GERHART, Genevra. 1974. The Russian's World: Life \& Language. New York: Harcourt, Brace, Jovanovich.

JUNG, Carl G. 1964, "Approaching the Unconscious". In Man and His Symbols, edited by Carl G. Jung.

LISIZIN, Francisco. 1984. "Secta religiosa molokan y la colonia rusa de Guadalupe, Ensenada, Baja California". Unpublished Ms. Research Library, San Diego Museum of Man.

LONG, Robert W. 1972. "Life and Times of José Matías Moreno". Doctoral dissertation, Westem University.

MACLENNAN, Hugh. 1945. Two Solitudes. New York: Duell, Sloan and Pearce.

MOHOFF, George. 1992a. The Russian Colony of Guadalupe: Molokans in Mexico. MS, United Molokan Christian Association Archives, 16222 E. Soriano Dr., Hacienda Heights, California.

- 1992b. Molokans in Mexico. Map.

MURANAKA, Therese Adams. 1987. "Los molokanos rusos de Baja California". Estudios Fronterizos 5(14):125-135.

NAULKO, V.I. 1978. "The Development of Inter-Ethnic Relations in the Ukraine". In Perspectives on Ethnicity, edited by Regina E. 
Holloman and Serghei A. Arutiunov, pp. 424-437. The Hague: Mouton.

PYSZCZYK, Heinz. 1990. "Some Thoughts on Garden Swings in a Northern Alberta Mennonite Community". Paper presented at the Annual Meeting of the Society for Historical Archaeology, Tucson. MS, Archaeological Survey of Alberta, Edmonton.

RONTGEN, Robert E. 1981. Marks on German, Bohemian and Austrian Porcelain, 1710 to the Present. Exton, Pennsylvania: Schiffer Publishing Ltd.

SPICER, Edward H. 1971. Persistent Cultural Systems. Science 174: 795-800.

- 1980. The Yaquis: A Cultural History. Tucson: University of Arizona Press,

STEPNIAK, a.k.a. Kravchinskii, Sergei Mikhailovich. 1888. The Russian Peasantry. New York: Harper and Brothers.

STEWARD, Julian. 1955. Theory of Culture Change. Urbana: University of Illinois.

STORY, Sidney Rochelle, 1960. "Spiritual Christian in Mexico: Profile of a Russian Village". MS, doctoral dissertation, Department of Anthropology, University of California, Los Angeles. An Arbor: University Microfilms International.

WOBST, Martin. 1977. Stylistic Behavior and Information Exchange. University of Michigan Museum of Anthropology, Anthropological Papers 61: 317-342,

\section{San Diego Union}

1905a Issue of August 26, 1905.

1905 b Issue of September 5, 1905.

1958a Issue of July 12, 1958, p. 1:4.

$1958 \mathrm{~b}$ Issue of July 13, 1958, p. 1:4-5.

1958c Issue of July 14, 1958, p. 1:6-7.

1958d Issue of July 15, 1958, p. 5:1-2.

$1958 \mathrm{e}$ Issue of July 16,1958 , p. 5:1-2.

1958f Issue of August 8, 1958, p. 5:1.

1959 Issue of July 11, 1959, p. 5:1. 